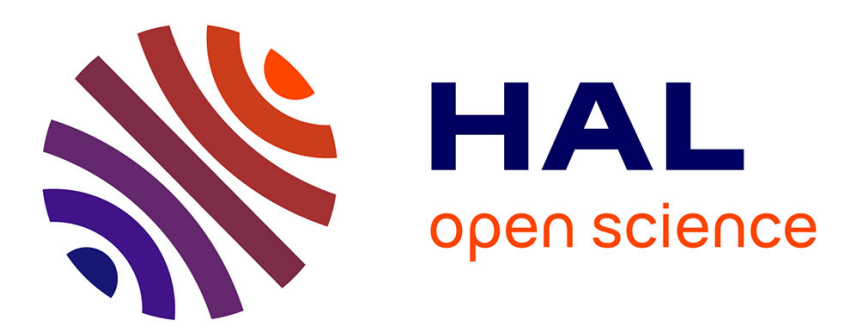

\title{
Myocardial Motion Estimation from Medical Images Using the Monogenic Signal
}

Martino Alessandrini, Adrian Basarab, Hervé Liebgott, Olivier Bernard

\section{To cite this version:}

Martino Alessandrini, Adrian Basarab, Hervé Liebgott, Olivier Bernard. Myocardial Motion Estimation from Medical Images Using the Monogenic Signal. IEEE Transactions on Image Processing, 2013, 22 (3), pp.1084-1095. 10.1109/TIP.2012.2226903 . hal-01121047v2

\section{HAL Id: hal-01121047 \\ https://hal.science/hal-01121047v2}

Submitted on 2 May 2013

HAL is a multi-disciplinary open access archive for the deposit and dissemination of scientific research documents, whether they are published or not. The documents may come from teaching and research institutions in France or abroad, or from public or private research centers.
L'archive ouverte pluridisciplinaire HAL, est destinée au dépôt et à la diffusion de documents scientifiques de niveau recherche, publiés ou non, émanant des établissements d'enseignement et de recherche français ou étrangers, des laboratoires publics ou privés. 


\title{
Myocardial Motion Estimation from Medical Images Using the Monogenic Signal
}

\author{
Martino Alessandrini, Adrian Basarab, Hervé Liebgott and Olivier Bernard
}

\begin{abstract}
We present a method for the analysis of heart motion from medical images. The algorithm exploits monogenic signal theory, recently introduced as an $\mathbf{N}$-dimensional generalization of the analytic signal. The displacement is computed locally by assuming the conservation of the monogenic phase over time. A local affine displacement model is considered to account for typical heart motions as contraction/expansion and shear. A coarse-to-fine B-spline scheme allows a robust and effective computation of the model's parameters and a pyramidal refinement scheme helps handle large motions. Robustness against noise is increased by replacing the standard point-wise computation of the monogenic orientation with a robust least-squares orientation estimate.

Given its general formulation, the algorithm is well suited for images from different modalities, in particular for those cases where time variant changes of local intensity invalidate the standard brightness constancy assumption. This study evaluates the method's feasibility on two emblematic cases: cardiac tagged magnetic resonance and cardiac ultrasound. In order to quantify the performance of the proposed method, we made use of realistic synthetic sequences from both modalities for which the benchmark motion was known. A comparison is presented with state-of-the-art methods for cardiac motion analysis. On the data considered, these conventional approaches are outperformed by the proposed algorithm. A recent global optical-flow estimation algorithm based on the monogenic curvature tensor is also considered in the comparison. With respect to the latter, the proposed framework provides, along with higher accuracy, superior robustness to noise and a considerably shorter computational time.
\end{abstract}

Index Terms-optical flow, monogenic signal, illumination changes, optimal window size, iterative refinement, cardiac ultrasound, tagged MRI.

\section{INTRODUCTION}

The monogenic signal has been recently introduced by Felsberg [1] as an extension of the analytic signal concept to multiple dimensions. Like the latter, the monogenic signal provides the local amplitude and local phase signal features. Additionally, it also contains information on the local orientation. These three local features are pointwise orthogonal, which means that they represent independent information: the local amplitude represents the local intensity or dynamics,

Martino Alessandrini, Hervé Liebgott and Olivier Bernard are with Centre de Recherche en Acquisition et Traitement de l'Image pour la Santé (CREATIS), Centre National de la Recherche Scientifique (CNRS) UMR 5220, Institut National de la Santé et de la Recherche Médicale (INSERM) U630, Université de Lyon, Institut National des Sciences Appliquées (INSA)Lyon, Villeurbanne, France. E-mail: mailto:martino.alessandrini@creatis.insalyon.frmartino.alessandrini@creatis.insa-lyon.fr

Adrian Basarab is with the Institut de Recherche en Informatique de Toulouse (IRIT), Centre National de la Recherche Scientifique (CNRS) UMR 5505, 118 Route de Narbonne, F-31062 Toulouse cedex 9, France. E-mail: mailto:adrian.basarab@irit.fradrian.basarab@irit.fr the local phase describes the local symmetry or grey value transition, and the local orientation describes the direction of the highest signal variance. Decoupling the local energy from the image structure, accounted for by phase and orientation, has made it possible to derive effective solutions to a number of image-processing problems, in particular when the more traditional pixel intensity cannot be considered as a reliable feature.

This situation is often encountered in medical imaging. In cardiac ultrasound, the local brightness varies over time due to the changes in the angle between the myocardial fibers and the direction of propagation of the acoustic beam or due to out-of-plane motions [2]. In magnetic resonance imaging (MRI), intensity variations stem from magnetic field inhomogeneities and scanner-related intensity artifacts [3]. In tagged-MRI (tMRI) [4], tags fade exponentially over time depending on the T1 relaxation time [5], [6]. Obviously, the same problems are encountered when contrast agents are used, such as in perfusion MRI [7] or when registration of images from different modalities, such as computed tomography (CT) and positron emission tomography (PET), is needed [8], [9].

These considerations explain the rapidly growing interest in monogenic signal analysis applied to medical imaging problems over the last few years. For example, one could cite successful applications of monogenic analysis in boundary detection [10], [11], segmentation [12], multi-modal registration [8], [9], ultrasound image compounding [13], multi-view image registration [14], wavelet filtering [15] and envelope detection [16].

In this context, the algorithm presented in this paper, addresses a further fundamental problem in the field of medical imaging, i.e. the estimation of myocardial motion. Assessment of myocardial elasticity and contractility is indeed essential in clinical practice to evaluate the degree of ischemia and infraction as well as for surgical planning [17], [18].

We compute the displacement estimate locally by assuming the conservation of the monogenic phase in lieu of traditional pixel brightness. The general formulation takes inspiration from the work of Felsberg in [19]; nevertheless, the novelties with respect to this study are manifold and substantial:

- Locally, the size of the image window is selected in order to have the most consistent motion estimate. This operation is fully automatic and computationally effective because of an adaptation of the B-spline multiresolution approach for the image moments computation proposed by Sühling et al. in [20], [21]. By doing so, a common source of error in local techniques, related to an inadequate choice of the window size, is avoided. 
- Felsberg's pure translation model is replaced with an affine model. The affine model, a part of translation, can account for rotation, expansion, compression and shear, and provides a realistic description of the motion patterns typical of the cardiac muscle [17]. Furthermore, as the first-order spatial derivatives of the displacement are also computed, it allows the direct computation of the cardiac strain, with no need for numerical differencing [18].

- The robustness to noise is improved by employing a least squares estimate of the monogenic orientation in place of the standard point-wise estimate [22].

- An incremental coarse-to-fine pyramidal scheme is used to refine the precision of the final estimate.

The general formulation makes the algorithm well suited for images from different modalities. In particular, this paper evaluates its performance on tagged magnetic resonance imaging (tMRI) [4] and cardiac ultrasound image sequences. In order to quantify performance, we made use of realistic synthetic sequences for both modalities, for which the benchmark motion was known. In each experiment, a comparison is presented with state-of-the-art methods in the related field. They include SinMod [23] for tMRI images and the Sühling [21] and Felsberg [19] algorithms for ultrasound. In both cases, the recent algorithm reported in Zang et al. [24] is also considered in the comparison. Indeed, due to the monogenic signal formulation at its base, it can be considered a possible competitor to the algorithm proposed herein.

The paper proceeds as follows. In Section II the monogenic signal theory is briefly summarized and the robust computation of the orientation is introduced. In Section III the proposed optical flow estimation algorithm is described. Section IV discusses some implementation details. In Section $\mathrm{V}$ the problem of cardiac motion analysis from tMRI and cardiac ultrasound is briefly summarized and the results are presented. Concluding remarks are left to Section VI.

\section{MonogeniC SignAl COMPUTATION}

The most practical aspects of the monogenic signal computation will be reviewed here. For further details, we address the interested reader to [1], [25] and to [22] for a more intuitive derivation.

The monogenic signal provides an extension of the standard analytic signal for multidimensional data. Although the theory is valid for a general number $N$ of dimensions $(N>1)$, we consider here the case at hand of $2 \mathrm{D}$ grayscale images $I: \Omega \rightarrow \mathbb{R}, \Omega \subset \mathbb{R}^{2}$.

The image model adopted in phase-based processing is [19], [26]:

$$
I(\mathbf{x})=A(\mathbf{x}) \cos (\varphi(\mathbf{x})),
$$

where $\mathbf{x}=[x, y]$ is the spatial coordinate vector, $A(\mathbf{x})$ is the local amplitude and $\varphi(\mathbf{x})$ is the local phase. Additionally, monogenic signal theory assumes a local intrinsic dimensionality one [19], [26], i.e., the local variations of $I$ are concentrated along a single direction, defined by the local orientation $\theta(\mathbf{x})$.

The monogenic signal computes the image features of amplitude, phase and orientation from the responses to three
2D spherical quadrature filters (SQFs) [1]. The SQFs consist of one even rotation invariant bandpass $b_{e}\left(\mathbf{x} ; \lambda_{0}\right)$ filter and two odd bandpass filters $b_{o 1}\left(\mathbf{x} ; \lambda_{0}\right)$ and $b_{o 2}\left(\mathbf{x} ; \lambda_{0}\right)$, where $\lambda_{0}$ is the filter wavelength, defined as the reciprocal of the normalized center frequency $f_{0}$. Note that in the following the dependency of the filter responses on the center frequency will be omitted for the sake of simplicity. The odd filters are computed from the Riesz transform of the even filter [1], [19]. In the frequency domain it is:

$$
B_{o 1}(\boldsymbol{\omega})=-\frac{j \omega_{x}}{|\boldsymbol{\omega}|} \cdot B_{e}(\boldsymbol{\omega}), \quad B_{o 2}(\boldsymbol{\omega})=-\frac{j \omega_{y}}{|\boldsymbol{\omega}|} \cdot B_{e}(\boldsymbol{\omega}),
$$

where capital letters denote the Fourier transformed quantities and $\boldsymbol{\omega}=\left[\omega_{x}, \omega_{y}\right]^{T}$ is the normalized angular frequency. Several SQF families have been employed in the literature: a comparison of the most popular ones is presented in [27]. A similar study is beyond the scope of this paper. Here, as recommended in [25], [28], the difference of Poisson (DoP) kernel is adopted:

$$
B_{e}(\boldsymbol{\omega})=\exp \left(-|\boldsymbol{\omega}| s_{1}\right)-\exp \left(-|\boldsymbol{\omega}| s_{2}\right),
$$

where $|\boldsymbol{\omega}|$ is the normalized angular frequency and $s_{1}$ and $s_{2}>s_{1}$ are two scale parameters. It can be shown that the wavelength is related to the two scales by:

$$
\lambda_{0}=2 \pi \frac{s_{1}-s_{2}}{\log \left(s_{1}\right)-\log \left(s_{2}\right)} .
$$

From the three filter responses, monogenic phase $\varphi(\mathbf{x})$, orientation $\theta(\mathbf{x})$ and amplitude $A(\mathbf{x})$ of $I$ are obtained as:

$$
\begin{aligned}
& \theta(\mathbf{x})=\arctan \left(\frac{q_{2}(\mathbf{x})}{q_{1}(\mathbf{x})}\right), \\
& \varphi(\mathbf{x})=\arctan \left(\frac{|\mathbf{q}(\mathbf{x})|}{p(\mathbf{x})}\right), \\
& A(\mathbf{x})=\sqrt{p^{2}(\mathbf{x})+|\mathbf{q}(\mathbf{x})|^{2}},
\end{aligned}
$$

where $p(\mathbf{x})=\left(I * b_{e}\right)(\mathbf{x}), q_{1}(\mathbf{x})=\left(I * b_{o 1}\right)(\mathbf{x}), q_{2}(\mathbf{x})=$ $\left(I * b_{o 2}\right)(\mathbf{x}), \mathbf{q}(\mathbf{x})=\left[q_{1}(\mathbf{x}), q_{2}(\mathbf{x})\right]^{T}$ and “*" denotes $2 \mathrm{D}$ convolution.

From the filter responses, the local frequency feature, defined as the derivative of the phase along $\mathbf{n}$, can also be computed as [19]:

$$
f \triangleq(\nabla \varphi)^{T} \cdot \mathbf{n}=\frac{p \nabla^{T} \mathbf{q}-\mathbf{q}^{T} \nabla p}{p^{2}+|\mathbf{q}|^{2}}
$$

where $\nabla=\left[\partial_{x}, \partial_{y}\right]^{T}$. Dependency on $\mathbf{x}$ is implied.

Monogenic phase and orientation can be conveniently combined in the phase vector $\mathbf{r}(\mathbf{x})=\left[r_{1}(\mathbf{x}), r_{2}(\mathbf{x})\right]=\varphi(\mathbf{x}) \cdot \mathbf{n}(\mathbf{x})$, with $\mathbf{n}(\mathbf{x})=[\cos (\theta(\mathbf{x})), \sin (\theta(\mathbf{x}))]^{T}[1]$, [19]. The dependency on $\mathbf{x}$ of all the aforementioned features will be omitted in the sequel unless necessary.

\section{A. Robust Orientation Computation}

In order to improve the robustness against image noise, in this study we replace the classical point-wise estimate of $\theta(5)$, with a robust least-squares estimate, inspired by the structure tensor formalism [29]. The scheme presented was proposed by Unser et al. in [22]. 
The least-squares orientation estimate is obtained by maximizing the directional Hilbert transform $\mathcal{H}_{\theta} I(\mathbf{x})$ averaged over a local neighborhood $v_{\sigma}$ :

$$
\bar{\theta}(\mathbf{x})=\arg \max _{\theta^{\prime} \in[-\pi, \pi]} \int_{\mathbb{R}^{2}} v_{\sigma}\left(\mathbf{x}^{\prime}-\mathbf{x}\right) \cdot\left|\mathcal{H}_{\theta^{\prime}} I\left(\mathbf{x}^{\prime}\right)\right|^{2} d \mathbf{x}^{\prime},
$$

where $v_{\sigma}$ corresponds here to a Gaussian kernel with variance $\sigma^{2}$ and the directional Hilbert transform is defined in the frequency domain as:

$$
\mathcal{H}_{\theta}(\boldsymbol{\omega})=\frac{\omega_{x} \cos (\theta)+\omega_{y} \sin (\theta)}{|\boldsymbol{\omega}|} .
$$

It is shown in [22] that (7) corresponds to the classical solution (5) if $v_{\sigma}(\mathbf{x})=\delta(\mathbf{x})$. The maximization problem (7) is solved by the eigenvector associated with the largest eigenvalue of the $2 \times 2$ matrix $\mathbf{T}(\mathbf{x})$, with entries:

$$
[\mathbf{T}(\mathbf{x})]_{n m}=\int_{\mathbb{R}^{2}} v_{\sigma}\left(\mathbf{x}^{\prime}-\mathbf{x}\right) q_{n}\left(\mathbf{x}^{\prime}\right) q_{m}\left(\mathbf{x}^{\prime}\right) d \mathbf{x}^{\prime},
$$

with $n, m=\{1,2\}$. The matrix $\mathbf{T}$ can be assimilated to a Riesz-transform counterpart of the standard structure tensor. The new estimate is then given by:

$$
\bar{\theta}(\mathbf{x})=\frac{1}{2} \arctan \left(\frac{2[\mathbf{T}(\mathbf{x})]_{12}}{\mathbf{T}[(\mathbf{x})]_{22}-\mathbf{T}[(\mathbf{x})]_{11}}\right) .
$$

Due to the averaging operation in (7), this alternative estimate is expected to be less sensitive to image noise than the traditional estimate. An example of this property is given in Fig. 1. We conclude this section by noting that this different orientation definition also affects the monogenic phase computation. In particular, the $|\mathbf{q}|$ term appearing in the second equation of (5) must now be replaced with $s(\mathbf{x})=$ $q_{1} \cos \bar{\theta}+q_{2} \sin \bar{\theta}$.

\section{Multiscale Optical Flow Computation from THE Monogenic Phase}

As in [19], the displacement field $\mathbf{d}(\mathbf{x})=\left[d_{1}(\mathbf{x}), d_{2}(\mathbf{x})\right]^{T}$ along $x$ and $y$ between two frames is estimated by replacing the traditional brightness constancy assumption with the more robust monogenic phase constancy assumption. This is conveniently expressed in terms of the monogenic phase vector as $\mathbf{r}(\mathbf{x}, t+1)=\mathbf{r}(\mathbf{x}-\mathbf{d}(\mathbf{x}), t)$. Assuming small displacements, the first-order Taylor expansion can be used $\mathbf{r}(\mathbf{x}-\mathbf{d}(\mathbf{x}), t) \approx \mathbf{r}(\mathbf{x}, t)-\mathbf{J}(\mathbf{x}, t) \cdot \mathbf{d}(\mathbf{x})$, where $\mathbf{J}$ is the Jacobian matrix of $\mathbf{r}$. Then, assuming all points translate of the same quantity $\mathbf{d}_{0}$ within a local window $w$ centered in $\mathbf{x}_{0}=\left[x_{0}, y_{0}\right]$, the following linear system of equations is obtained:

$$
\begin{gathered}
\langle\mathbf{J}\rangle_{w} \mathbf{d}_{0}=-\left\langle\mathbf{r}_{t}\right\rangle_{w}, \\
\mathbf{J}(\mathbf{x}, t)=\left[\begin{array}{ll}
r_{1 x}(\mathbf{x}, t) & r_{1 y}(\mathbf{x}, t) \\
r_{2 x}(\mathbf{x}, t) & r_{2 y}(\mathbf{x}, t)
\end{array}\right],
\end{gathered}
$$

where $\mathbf{r}_{t}(\mathbf{x}, t)=\left[r_{1 t}(\mathbf{x}, t), r_{2 t}(\mathbf{x}, t)\right]$ denotes the time derivative of $\mathbf{r}$, approximated as $\mathbf{r}(\mathbf{x}, t+1)-\mathbf{r}(\mathbf{x}, t),\langle\mathbf{v}\rangle_{w}$ denotes the weighted average $\int_{\Omega} w\left(\mathbf{x}-\mathbf{x}_{0}\right) \mathbf{v}(\mathbf{x}) d \mathbf{x}$ and $r_{i k}=\partial_{k} r_{i}$. Dependency on $(\mathbf{x}, t)$ will be omitted in the following.

Assuming a 1D structure [19], $\mathbf{J}$ must have rank one. It can be shown that its only eigenvalue corresponds to the monogenic frequency $f$ in (6) while the associated eigenvector

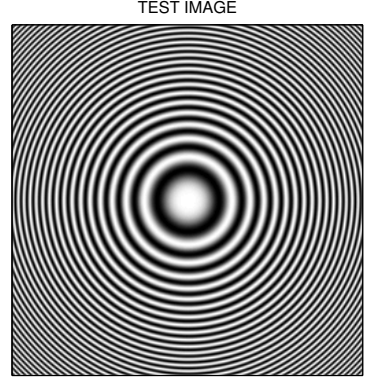

(a)

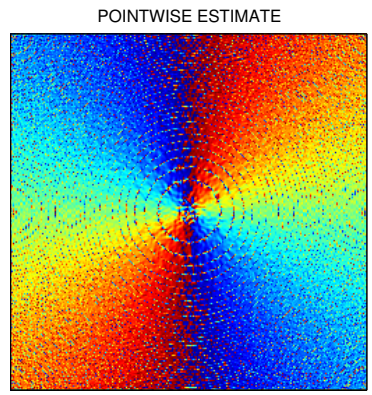

(c)

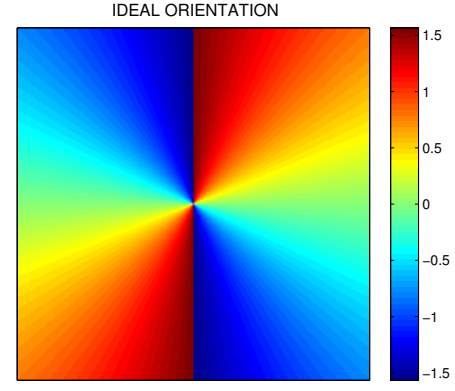

(b)

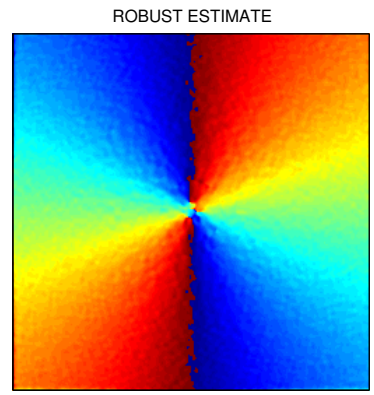

(d)
Fig. 1. Monogenic orientation estimate in the presence of noise. (a) noisefree test image containing a full 360-degree range of orientations. (b) ideal orientation. (c-d) pointwise and robust $(\sigma=2)$ estimates in the presence of image noise $(20 \mathrm{~dB})$. Mean square error of the estimate is $1.2 \mathrm{E}-2$ for $(\mathrm{c})$ and $2.7 \mathrm{E}-4$ for $(\mathrm{d})$. Phases are wrapped in the $[-\pi / 2, \pi / 2]$ interval.

is $\mathbf{n}=[\cos (\theta), \sin (\theta)]^{T}$ [22], [19], this leads to the expression [22], [19]:

$$
\mathbf{J}=f \mathbf{n} \mathbf{n}^{T}=f\left[\begin{array}{cc}
\cos ^{2}(\theta) & \sin (\theta) \cos (\theta) \\
\sin (\theta) \cos (\theta) & \sin ^{2}(\theta)
\end{array}\right] .
$$

The term $\mathbf{r}_{t}$ is computed from the SQFs responses as [19]:

$$
\mathbf{r}_{t}=\frac{p_{\mathrm{t}} \mathbf{q}_{\mathrm{t}+1}-\mathbf{q}_{\mathrm{t}} p_{\mathrm{t}+1}}{\left|p_{\mathrm{t}} \mathbf{q}_{\mathrm{t}+1}-\mathbf{q}_{\mathrm{t}} p_{\mathrm{t}+1}\right|} \arctan \left(\frac{\left|p_{\mathrm{t}} \mathbf{q}_{\mathrm{t}+1}-\mathbf{q}_{\mathrm{t}} p_{\mathrm{t}+1}\right|}{p_{\mathrm{t}} p_{\mathrm{t}+1}+\mathbf{q}_{\mathrm{t}}^{T} \mathbf{q}_{\mathrm{t}+1}}\right)
$$

where subscripts " $t$ " and " $t+1$ " denote the time instant.

We conclude by noting that (11) represents the monogenic phase counterpart of the popular Lucas \& Kanade algorithm [30], where the matrix $\mathbf{J}$ replaces the image structure tensor.

\section{A. Affine model}

Clearly, the simple translation model employed by Felsberg is too restrictive in a general context. Also, its validity is heavily dependent on the choice of the size of $w$. The solution we propose is to replace the constant motion assumption with a more general model, such as the affine model [31], [32]. A part of translations, this accounts for rotation, expansion, compression and shear. In the context of this paper, the affine model is of major interest because it provides a realistic description of the motion patterns of the cardiac muscle [17]. A further relevant point is that, as the first-order spatial derivatives of the displacement are also computed, the Lagrangian strain tensor can be directly obtained from the latter, with no need for further numerical differencing. The local analysis of cardiac contractility is indeed fundamental in the diagnosis of pathological situations such as ischemia [18], [33]. 
Considering for simplicity a window $w$ centered at $\left(x_{0}, y_{0}\right)=(0,0)$, the affine model is written:

$$
\mathbf{d}(\mathbf{x})=\mathbf{A}(\mathbf{x}) \mathbf{u}, \quad \mathbf{A}=\left[\begin{array}{llllll}
1 & 0 & x & y & 0 & 0 \\
0 & 1 & 0 & 0 & x & y
\end{array}\right],
$$

where $\mathbf{u}=\left[d_{10}, d_{20}, d_{1 x}, d_{1 y}, d_{2 x}, d_{2 y}\right]^{T}$ is the new unknown vector: $d_{10}$ and $d_{20}$ correspond to the translation of the window center and $d_{i k}=\partial_{k} d_{i}$.

Plugging (14) into (11) leads to an underdetermined system of equations. The solution is obtained by pre-multiplying both terms by $\mathbf{A}^{T}$, hence:

$$
\langle\mathbf{M}\rangle_{w} \mathbf{u}=\langle\mathbf{b}\rangle_{w}, \quad \mathbf{M}=\mathbf{A}^{T} \mathbf{J} \mathbf{A}, \quad \mathbf{b}=-\mathbf{A}^{T} \mathbf{r}_{t} .
$$

Equation (15) represents the proposed monogenic phase version of the Lucas \& Kanade algorithm with affine parametrization of the displacement [21].

It can be shown that the entries of $\mathbf{M}$ and $\mathbf{b}$ are the local moments of orders zero to two of the spatial and temporal derivatives of $r_{1}$ and $r_{2}$ :

$$
\begin{aligned}
\mathbf{M} & =\left[\begin{array}{cccccc}
r_{1 x} & r_{1 y} & x r_{1 x} & y r_{1 x} & x r_{1 y} & y r_{1 y} \\
r_{2 x} & r_{2 y} & x r_{2 x} & y r_{2 x} & x r_{2 y} & y r_{2 y} \\
x r_{1 x} & x r_{1 y} & x^{2} r_{1 x} & x y r_{1 x} & x^{2} r_{1 y} & x y r_{1 y} \\
y r_{1 x} & y r_{1 y} & x y r_{1 x} & y^{2} r_{1 x} & x y r_{1 y} & y^{2} r_{1 y} \\
x r_{2 x} & x r_{2 y} & x^{2} r_{2 x} & x y r_{2 x} & x^{2} r_{2 y} & x y r_{2 y} \\
y r_{2 x} & y r_{2 y} & x y r_{2 x} & y^{2} r_{2 x} & x y r_{2 y} & y^{2} r_{2 y}
\end{array}\right], \\
\mathbf{b} & =-\left[\begin{array}{llllll}
r_{1 t} & r_{2 t} & x r_{1 t} & x r_{2 t} & y r_{1 t} & y r_{2 t}
\end{array}\right] .
\end{aligned}
$$

Note that, according to (12), it is $r_{1 x}=\cos ^{2}(\theta), r_{2 y}=$ $\sin ^{2}(\theta)$ and $r_{2 x}=r_{1 y}=\sin (\theta) \cos (\theta)$.

\section{B. Multiscale choice of window size.}

The choice of the window size is a tedious issue connected with local techniques: the assumed motion model (translational or affine) may not hold when the window is too big, otherwise, the adoption of an excessively small window may result in the well known aperture problem [34]. To circumvent this issue, in [20], [21] Sühling et al. proposed a multiscale strategy for locally choosing the most consistent window size. This is based on the possibility of computing the image moments, i.e., the entries of the system matrix $M$ and the vector $b$ in (16), at multiple scales, by using an efficient B-spline coarse-to-fine strategy.

In particular, they are obtained from window functions $w$ that are progressively scaled and subsampled by a factor 2 in each dimension. More precisely, at scale $j$, the window $w^{j}\left(\mathbf{x}-\mathbf{x}_{0}\right)=w\left(\left(\mathbf{x}-2^{j} \mathbf{x}_{0}\right) / 2^{j}\right)$ is employed, where $w$ is written as the separable product of two B-spline functions.

By doing so, at each scale $J_{f} \leq j \leq J_{c}\left(J_{f} \geq 0\right)$ a solution $\mathbf{u}^{j}$ can be computed. Among the scales considered, the $\mathbf{u}^{j}$ producing the smallest residual error $\left.\left\|\mathbf{M} \mathbf{u}^{j}-\mathbf{b}\right\|\right|_{\ell 2} /|w|_{\ell 1}$ is retained as the final displacement estimate. Whenever necessary, bi-cubic interpolation is employed to obtain a dense motion field. With this strategy, the scale providing the most consistent motion estimate is selected.

\section{Iterative displacement refinement}

The hypothesis of small displacements employed in differential techniques may be inadequate whenever the displacement is substantial or the image intensity profile is non-linear. A possible way to deal with this limitation is to implement a form of Gauss-Newton optimization: the current estimate is used to undo the motion, and then the estimator is reapplied to the warped images to find the residual displacement [24], [31], [35]. When applied iteratively, this procedure can improve the estimation accuracy considerably.

We employed the aforementioned refinement scheme in the algorithm presented. In particular, we found it to be particularly effective when the degree of detail in the monogenic phase image progressively increases between subsequent iterations. In practice, this is established by suitably tuning the center frequency $f_{0}$ of the SQF bank. By doing so, the coarsest image is first employed to determine a rough estimate of the displacement. This estimate is then adjusted on the finer detail data, obtained from an higher value of center frequency.

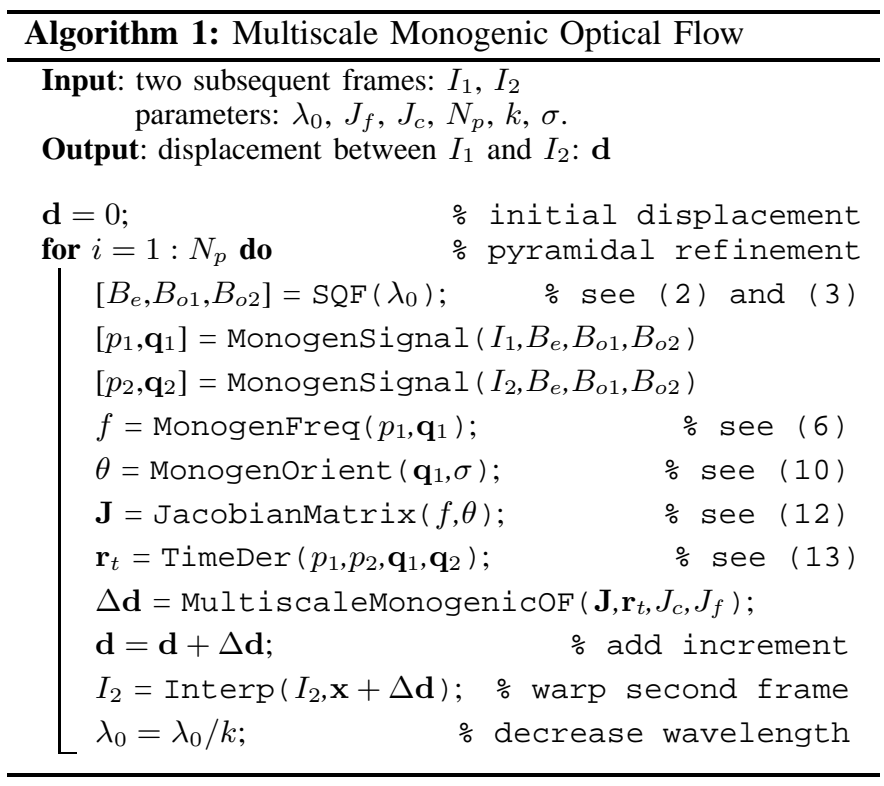

\section{IMPLEMENTATION DETAILS}

The pseudo-code of the proposed algorithm is presented in Algorithm 1. The pyramidal refinement scheme of Section III-C was implemented by decreasing the filter wavelength $\lambda_{0}$ by a factor $k=1.5$ at each iteration. The number of iterations $N_{p}$ and the starting wavelength value have been optimized in each of the experiments described in the next session.

The multiscale window choice was implemented by considering fifth-order B-splines and scales $j=\{2,3,4,5\}$. We note that at scale $j$ the motion is computed on square windows with sides $5 \cdot 2^{j}-1$, with a spacing of $2^{j}$ pixels between neighboring estimates. A value $\sigma=2$ was used for the robust computation of the monogenic orientation.

The proposed algorithm has been implemented in MATLAB (R2011b, The Math-Works, Natick, MA). The code is made freely available at http://www.creatis.insa-lyon.fr/ustagging/code. 


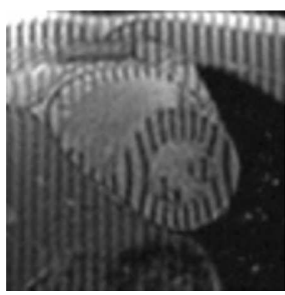

(a)

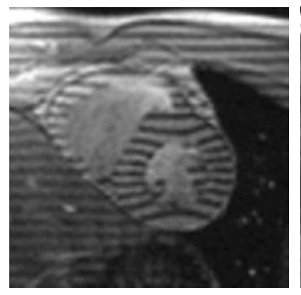

(b)

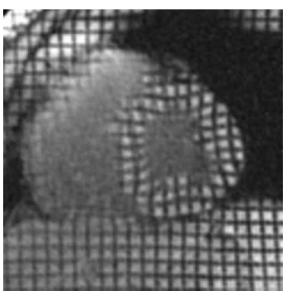

(c)
Fig. 2. (a) Vertical, (b) horizontal and (c) grid tags. Images from [6].

\section{RESUlts}

The algorithm was tested on realistic simulated cardiac ultrasound and tagged cardiac MRI (tMRI) image sequences for which the benchmark motion was known. In each case, a comparison will be presented with state-of-the art algorithms for cardiac motion estimation and with the algorithm of Zang et al. [24], which, to the best of our knowledge, is the most closely related work to the study presented in this paper. The Zang algorithm is briefly summarized in Appendix A.

Concerning performance assessment, the most commonly used measurement in the literature is the angular error [36]. Nevertheless, this metric has several shortcomings. At first, due to the arbitrary scaling constant (1.0) used to avoid the divide-by-zero problem, it penalizes small displacements more than large ones. Second, symmetrical deviations of estimated vectors from the true value result in different error values.

For these reasons, we employ here the less conventional but more appropriate endpoint error (EE) [37], [38]:

$$
E E=\|\mathbf{d}-\overline{\mathbf{d}}\|_{2},
$$

where $\mathbf{d}$ denotes the estimated displacement and $\overline{\mathbf{d}}$ the benchmark displacement.

\section{A. MRI Tagging}

1) Background: Tagged MRI is currently the gold-standard technique for quantification of myocardial contractility in vivo [39], [23]. With this technique, cardiac tissue is marked with magnetically saturated tagging lines or grids (cf. Fig. 2) that deform with the underlying tissue during the cardiac cycle, thus providing details on the myocardial motion. With time elapsing, the grid loses contrast and sharpness (cf. Fig. 3(a)(c)). This is the reason why state-of-the-art techniques for the estimation of myocardial motion from tMRI sequences exploit the image phase rather than the less trustworthy pixel intensity. The popular algorithms HARP (harmonic phase) [39] and SinMod (sine-wave modeling) [23] belong to this family of methods. In particular, the latter was shown to outperform HARP in [23].

Both the aforementioned algorithms are derived from modeling the tMRI image as the superposition of monochromatic plane waves:

$$
I(\mathbf{x}) \approx A(\mathbf{x}) \cos \left(\boldsymbol{\omega}_{0}^{T} \mathbf{x}\right),
$$

where $\omega_{0}$ is fixed given tags direction and spacing. The displacement is then computed in the Fourier domain from

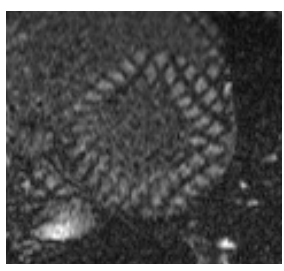

(a)

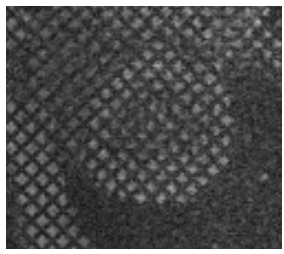

(d)

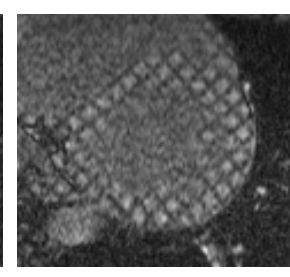

(b)

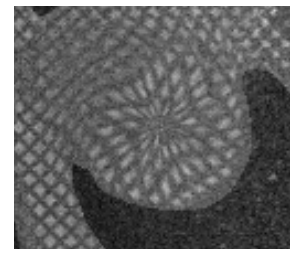

(e)

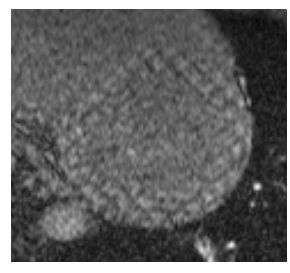

(c)

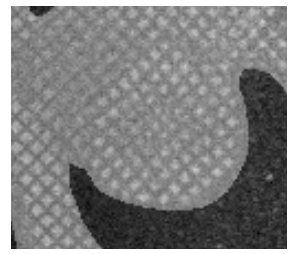

(f)
Fig. 3. Tags fading effect on a real tMRI sequence (a)-(c) and on a simulated one (d)-(f).

the responses of a set of bandpass directional filters tuned accordingly to $\boldsymbol{\omega}_{0}$. More specifically, while HARP [39] employs a phase-based disparity measure similar to the one by Fleet and Jepson [40], SinMod estimates the displacement based on an analytical expression for the cross-power spectrum of two subsequent frames [23].

It is interesting to observe (18) in relation with the work presented here. At first, that model directly satisfies the assumption of 1D local structures, at the base of the monogenic signal analysis. This makes the monogenic signal a promising tool for the study of tMRI sequences. To our knowledge, this is the first study investigating this possibility. Second, (18) can be readily obtained from (1) by including the first-order phase expression used in Section III. This reveals that on tMRI images the assumption of small displacements is no longer required. The upper-limit for the displacement is now given by one-half of the tag spacing, beyond which the motion estimation problem becomes undetermined.

2) Motion Estimation Results: The proposed algorithm is compared with SinMod, available in the InTag plugin for OsiriX ${ }^{1}$. The evaluation was made on synthetic tMRI sequences, generated with the ASSESS software [41]. The synthetic motion is established on the basis of a 2D analytical model taking typical contraction, relaxation, torsion and thickening of the cardiac muscle into account [42]. The characteristic tag-fading effect, not considered in ASSESS, was also taken into account in this study, as shown in Fig. 3(d)-(f). The effect was obtained by adjusting the image's histogram limits on each frame so as to match those of a real sequence taken as a template. The algorithm of Zang et al. [24] was also considered in the comparison.

The results obtained on nine simulated sequences are summarized in Table I. For each algorithm the parameters were optimized to return the smallest average error on the sequence D30R20T01P0F20. For the proposed algorithm, these values were $\lambda_{0}=4$ for the initial wavelength and $N_{p}=5$ for the number of refinement steps. For the Zang algorithm,

\footnotetext{
${ }^{1}$ http://www.creatis.insa-lyon.fr/inTag/
} 
TABLE I

ENDPOINT ERROR $(\mu \pm \sigma)$ IN PIXELS ON 9 SIMULATED SEQUENCES.

\begin{tabular}{lccc}
\hline SEQUENCE & \multicolumn{3}{c}{ ALGORITHM } \\
\hline & Proposed & SinMod & Zang \\
\hline D30 & $\mathbf{0 . 1 5 2} \pm \mathbf{0 . 1 2 1}$ & $0.215 \pm 0.145$ & $0.163 \pm 0.137$ \\
D30F20 & $\mathbf{0 . 0 8 2} \pm \mathbf{0 . 0 7 2}$ & $0.128 \pm 0.112$ & $0.087 \pm 0.079$ \\
D30R10T01P0 & $\mathbf{0 . 2 6 4} \pm \mathbf{0 . 1 4 9}$ & $0.363 \pm 0.199$ & $0.303 \pm 0.202$ \\
D30R20T01P0 & $\mathbf{0 . 4 6 2} \pm \mathbf{0 . 2 3 9}$ & $0.970 \pm 1.129$ & $0.531 \pm 0.328$ \\
D30R20T01P0F20 & $\mathbf{0 . 2 0 9} \pm \mathbf{0 . 1 3 9}$ & $0.344 \pm 0.224$ & $0.224 \pm 0.174$ \\
D30R20T01P3 & $\mathbf{0 . 4 1 9} \pm \mathbf{0 . 2 2 8}$ & $0.911 \pm 1.099$ & $0.461 \pm 0.301$ \\
R20F20 & $\mathbf{0 . 2 4 4} \pm \mathbf{0 . 1 6 4}$ & $0.416 \pm 0.264$ & $0.247 \pm 0.191$ \\
R10 & $\mathbf{0 . 1 6 1} \pm \mathbf{0 . 0 8 7}$ & $0.220 \pm 0.090$ & $0.164 \pm 0.104$ \\
R20 & $\mathbf{0 . 1 0 4} \pm \mathbf{0 . 0 7 2}$ & $0.174 \pm 0.122$ & $0.124 \pm 0.079$ \\
\hline
\end{tabular}

the values were $\alpha=0.2$ for the weight between the data and the smoothness term, $\gamma=0.1$ for the weight between the monogenic signal and the monogenic curvature and a variance $\rho^{2}$ of 2 pixels for the Gaussian localizing window (see Appendix A for a clearer understanding of the parameters' meaning). A multi-resolution refinement scheme was also employed [24] with four levels. SinMod required the tags type (grid), direction $\left(45^{\circ}\right)$ and spacing (six pixels). The name of each sequence reflects the values of the parameters used for its generation, namely: contraction/expansion (D), rotation (R), thickening (T), frame-rate (F) and healthy (P0) or pathological (P3) state. Greater detail on their meaning can be found in [42].

These results show that the proposed algorithm systematically returns the estimate with the smallest mean value and variance, which is a proof of precision and reliability. While the improvement with respect to SinMod is evident, the improvement with respect to the Zang algorithm is less pronounced. Nevertheless, the differences among all the algorithms were found to be statistically significant $(p<0.0001)$ for all sequences using the Friedman rank test $(\alpha=0.05)$ in conjunction with the post-hoc test proposed by Daniel [43], as suggested in [44]. In order to avoid correlations among samples, we suitably subsample the error images prior to the statistical analysis.

A clearer understanding of the algorithm's performance is provided by Fig. 4 where the error dispersion on two of the simulated sequences is represented for the three algorithms considered. The sequences were considered in order to present two different kinds of motions, specifically pure rotation (a) and pure contraction/expansion (b). In both cases, the proposed algorithm and Zang's algorithm outperform SinMod. It is also clear how the proposed algorithm provides better estimates than Zang's in the first part of the sequence, i.e. when the displacements are greater, while, in the final part, the two estimates are almost equivalent.

To better appreciate the difference in performance, it is useful to analyze the local behavior of each algorithm. This is represented in Fig. 5, where the error images obtained on the 4-th frame of the two sequences considered above is displayed. At that instant, the displacement reaches the maximum average value and the greatest spatial variation in both cases: in the

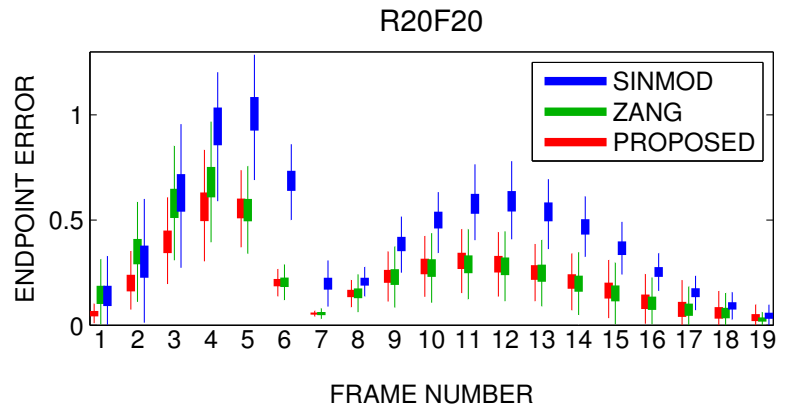

(a)

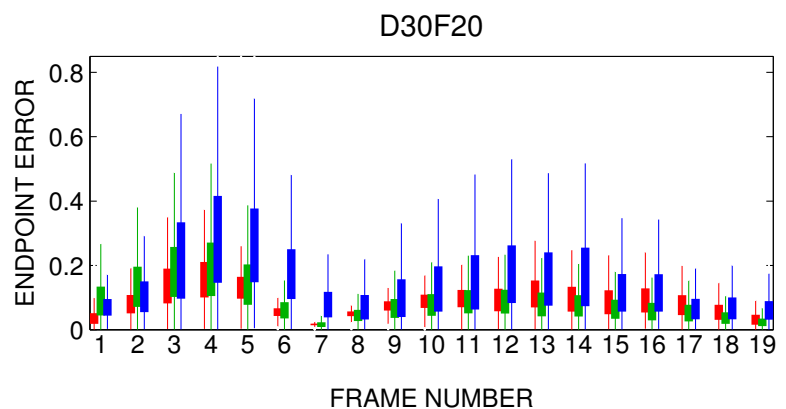

(b)

Fig. 4. Boxplot of the errors for R20F20 in (a) and D30F20 in (b). The center of each box represents the median while the body extends from the 25-th to the 75-th percentile.

first case (first row in the Figure) the angular velocity decreases linearly, passing from the endocardial to the epicardial contour; in the second (second row in the figure) the radial contraction is null on the epicardium and maximal on the endocardium.

From the comparison between Fig. 5(c)-(g) and Fig. 5(d)(h) it is clear how the Zang algorithm suffers more from these gradients of velocity than the proposed algorithm. This is a consequence of its global nature. Indeed, this method imposes a constraint on the gradient of the motion field that turns out to be inadequate when the entity of the displacement varies rapidly inside the image. At this point, it is important to remember that these results correspond to the optimal parameters' configuration. In particular, smaller values of the smoothness weight $\alpha$, which could tentatively be employed in order to avoid over-regularization effects, lead instead to larger errors. For example, a reduction of $\alpha$ from the optimal 0.2 to 0.05 leads to an increase in the endpoint error from 0.45 to 0.68 pixels. As shown by the previous results, SinMod is outperformed by both methods.

More generally, Zang's algorithm appears to involve excessively rigid priors on the displacement model, which makes it unsuitable to dealing with more complex and inhomogeneous motion patterns. In contrast, the proposed algorithm does not imply any hypothesis on the motion field, and therefore it can handle similar situations with superior flexibility.

The sensitivity to noise was also evaluated. To this end, we contaminated the frames of sequence R20F20 with additive Rician noise [5]. Fig. 6 reports the endpoint error variation due to noise, i.e. the value $\left|E E_{n}-E E_{\text {ref }}\right|$, where $E E_{\text {ref }}$ is the average endpoint error measured in the noise-free case (cf. 


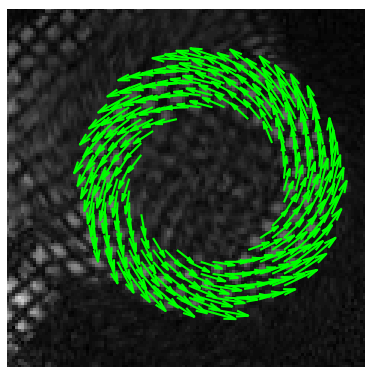

(a)

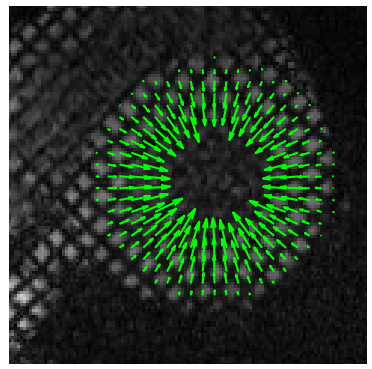

(e)

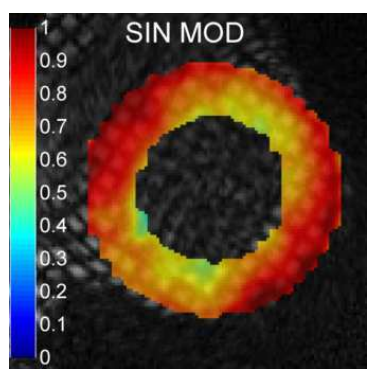

(b)

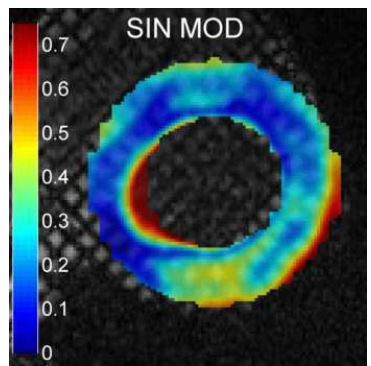

(f)

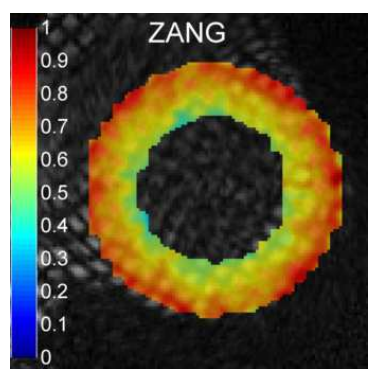

(c)

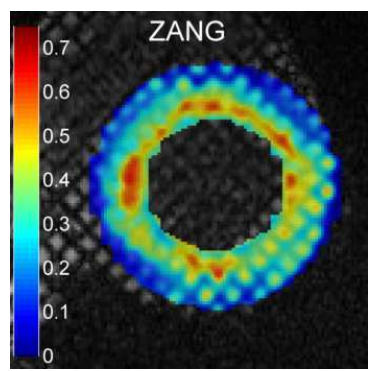

(g)

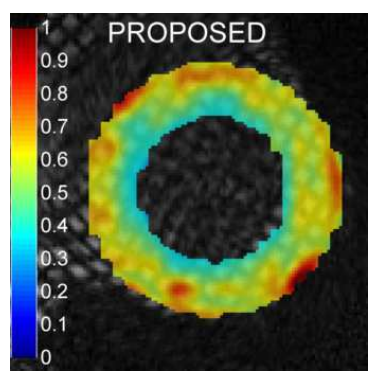

(d)

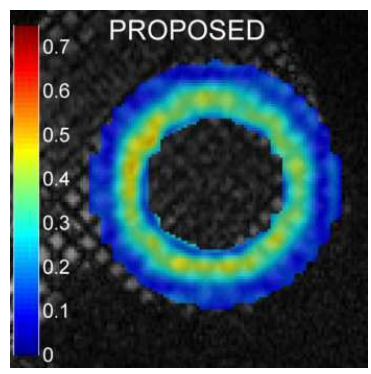

(h)

Fig. 5. Error map for the 4-th frame of R20F20 (first row) and D30F20 (second row). The green arrows in (a) and (e) denote the benchmark field.

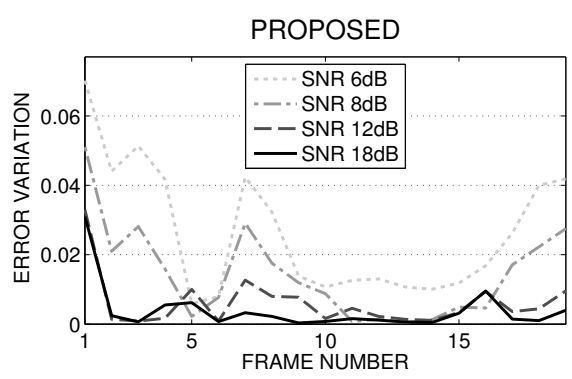

(a)

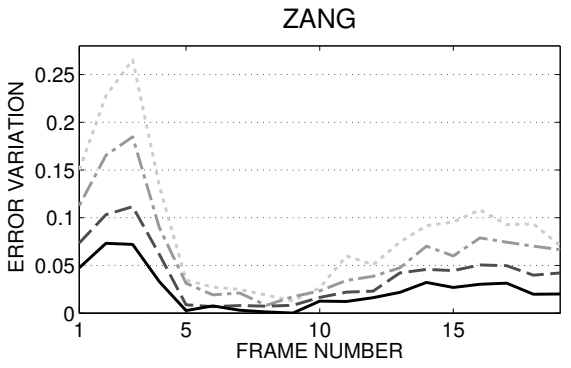

(b)

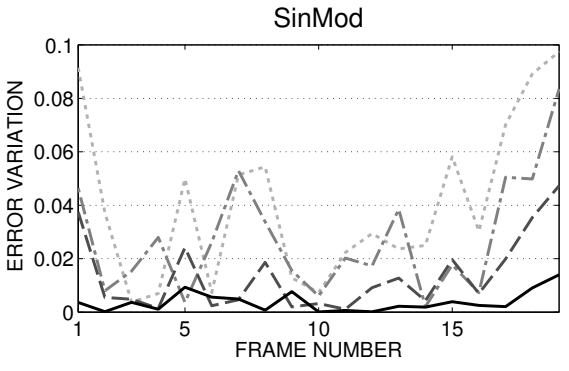

(c)

Fig. 6. Sensitivity to noise of proposed algorithm in (a), Zang's algorithm in (b), SinMod in (c)

Fig. 4), while $E E_{n}$ is the value in the presence of noise. The results are based on 15 independent noise realizations. While the performance of the Zang algorithm decreases considerably, especially for large motions, the performance of the proposed algorithm remains virtually unchanged. The good robustness against noise stems from two factors: the multiscale window choice of Section III-B and the robust monogenic orientation of Section II-A. The first guarantees that the integration scale is optimized locally so as to minimize the noise effect on the velocity determination, while the second ensures a more robust computation of the monogenic features. We also note that sensitivity to noise is a known drawback of global techniques as compared to local techniques [45]. SinMod also shows better noise robustness as compared to the Zang algorithm. Nevertheless, it should be noted that SinMod also returned the worst results in terms of accuracy.

Here we note that the computation of the monogenic signal involves pre-filtering the data, and this can produce some noise suppression. Nevertheless, this fact does not explain the superiority with respect to Zang's algorithm given that the latter makes use of the same set of SQF filters that we employ in the proposed method.

A further fundamental point concerns computational time. For the optimal parameters' configuration, it was $0.55 \mathrm{~s} / \mathrm{image}$ for the proposed algorithm (image size, $256 \times 256$ pixels $^{2}$ ) and 17 s/image for Zang's algorithm. Both these values refer to MATLAB implementations executed on a desktop PC with a $3.47 \mathrm{GHz}$ Intel Xeon X5690 processor, $12 \mathrm{~Gb}$ of RAM and running Windows 7. Although unoptimized for definition, given that MATLAB was used, these results give a clear vision on the relation between the complexity of the two algorithms. The increased computational burden of the Zang algorithm is readily explained by its global formulation, demanding the employment of iterative optimization routines, cf. Appendix A. On the contrary, the proposed algorithm reaches a subsecond speed with its efficient B-spline formalism (even in this unoptimized version). It is worth pointing out that fast computation is primal as far as medical imaging is concerned.

Finally, the feasibility of the algorithm presented in a clinical setting was qualitatively assessed by considering two real acquisitions. The first came from a healthy subject (Siemens MAGNETOM Avanto 1.5T, $6 \mathrm{~mm}$ tag-spacing, $0^{\circ}$ 

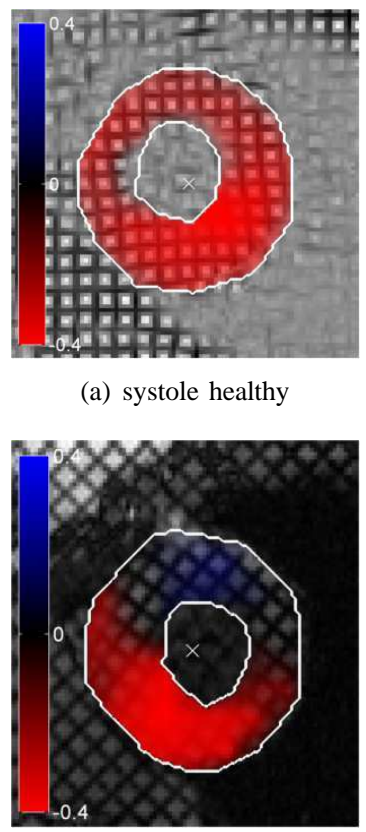

(c) systole post infarct (a) systole healthy

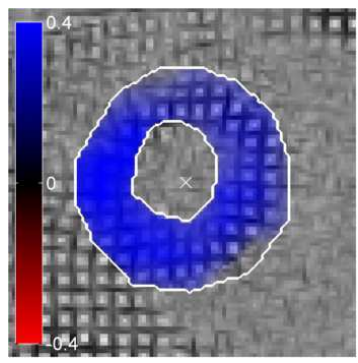

(b) diastole healthy

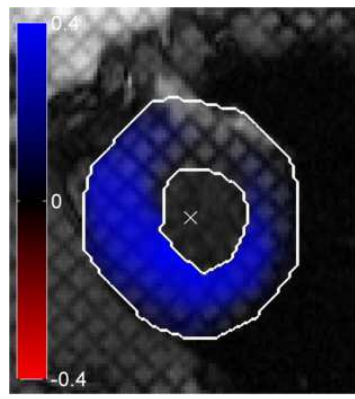

(d) diastole post infarct
Fig. 7. Color encoding of the radial component of the estimated displacement. Red color encodes inward motion and blue color outward one. No color denotes no motion. The displacement value is expressed in pixel. In (a) and (b) are presented the results on a systolic and diastolic frame on an healthy subject. In (c) and (d) are presented the results on a systolic and diastolic frame on a post-infarction subject.

tag-orientation), the second from a patient who underwent inferior cardiac infarction due to the occlusion of the left anterior descending artery (LAD). This latter acquisition refers to two days after reperfusion (Siemens MAGNETOM Avanto $1.5 \mathrm{~T}, 6 \mathrm{~mm}$ tag-spacing, $45^{\circ}$ tag-orientation). A qualitative representation of the results is given in Fig. 7. The color map superimposed on the tMRI image encodes the radial component of the estimated displacement computed with respect to the center of the myocardium, represented by a white cross. Red and blue denote inward and outward displacement, respectively.

The first line of figures corresponds to a systolic and diastolic frame on the healthy subject: the estimated displacement reflects the physiological contraction and dilatation of the left ventricle in these two phases of the heart cycle. In contrast, on the post-infarct patient, the color notation reflects the reduced mobility of the heart regions involved in the infarction. More than that, Fig. 7(c) demonstrates a dyskinetic behavior, represented by an non physiological outward motion during systole [21].

In the experiments illustrated in Fig. 7, the heart mask was drawn manually by a cardiologist and the center point was computed as its center of mass. Several ways for automatizing myocardium tracking on tMRI sequences have been proposed in the literature and could be employed here in lieu of manual contouring. Reviewing them is beyond the scope of this paper.

Clearly, the evaluation proposed above is far from being an exhaustive clinical evaluation of the proposed algorithm. Still, it gives insights into the meaningfulness of the estimates it returns. A deeper evaluation on diagnostic cases is left to further studies.

We conclude this section by noting that, even though the model (18) is adequate for line-tags, otherwise, in the case of grid-tags, a second wave roughly perpendicular to the first should be included in the image model. This would suggest investigating the use of 2D extensions of the monogenic signal. In particular the signal multi-vector [28] shows excellent fit with the grid-tag image model. Similar considerations deserve to be investigated more in depth in future studies. Nonetheless, the results presented here show that, even in the grid-tag case, the monogenic-phase-based algorithm presented still produces relevant estimates.

\section{B. Cardiac Ultrasound}

1) Background: Quantitative analysis of cardiac ultrasound sequences can provide important mechanical measurements such as muscle strain and twist, wall thickness and ejection fraction [18]. Compared to MRI, medical ultrasound has a higher spatio-temporal resolution, requires no infrastructures, low budgets and involves no discomfort for the patients. For these reasons it is currently the most widespread medical imaging exam [46]. These factors explain the high clinical interest in the development of tools for the determination of cardiac function from cardiac ultrasound images [18].

While tissue Doppler offers a powerful instrument to evaluate cardiac deformation [47], it suffers from the major limitation that only the velocity component in the direction of the ultrasound beam can be determined. This has motivated a growing interest in the development of non-Doppler techniques. They include speckle-tracking [48], frame-to-frame [49] or group-wise elastic registration [33] and optical flow [21]. In particular, the algorithm of Sühling et al. [21] achieves an excellent compromise between accuracy and computational complexity. Moreover, its clinical feasibility has been attested in thorough studies [17].

The Sühling algorithm improves the Lucas \& Kanade [30] formalism by including the multiscale window choice strategy of Section III-B. As in [30], motion is computed on the basis of the brightness conservation between subsequent frames. Nevertheless, as mentioned in the introduction, this can be a misleading assumption as far as cardiac ultrasound is concerned. This is also proved by the increasing interest in phase-based solutions [11], [14].

The following compares the proposed multiscale monogenic optical-flow algorithm presented in this paper, the Sühling algorithm, the Zang algorithm and the Felsberg algorithm, which has been recently applied to medical ultrasound in [50].

2) Motion Estimation Results: In order to provide a quantitative evaluation of the algorithms considered, we use synthetic echocardiographic sequences. The simulation framework is described in [51]. The simulated sequences along with the benchmark fields are available for download at http://www.creatis.insa-lyon.fr/us-tagging/news. In this study, we assessed two simulated sequences: one Short Axis (SAx) and one Apical 4 Chambers (A4C). These are two of the most frequently adopted orientations in the clinical procedure [52].

A representation of the estimated motion fields with the proposed algorithm is given in Fig. 8. These fields show how 


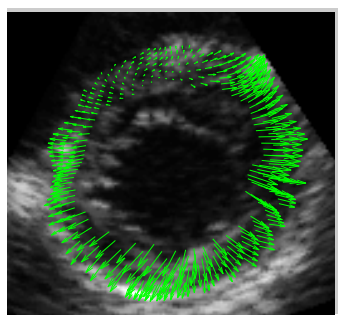

(a)

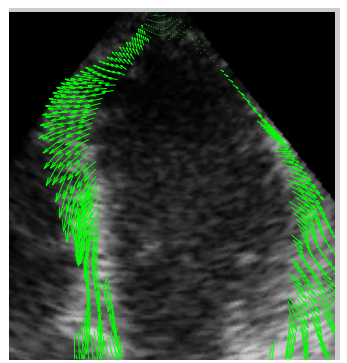

(c)

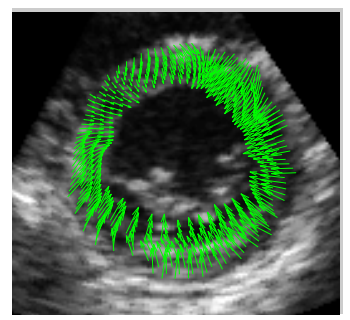

(b)

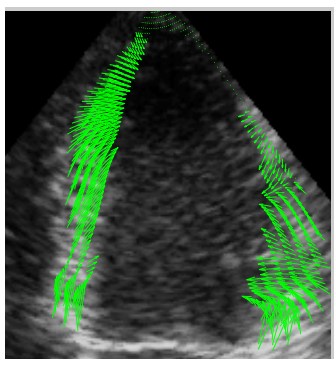

(d)
Fig. 8. (a),(b) Diastolic and systolic frames from a synthetic short axis sequence. The motion estimated with the proposed algorithm is superimposed as green arrows. (c),(d) Diastolic and systolic frames from a synthetic apical four chambers sequence.

the estimates are qualitatively consistent with physiological cardiac motion: indeed the motion vectors point inward during systole and outward during diastole.

TABLE II

ENDPOINT ERROR $(\mu \pm \sigma)$

\begin{tabular}{lcc}
\hline ALGORITHM & \multicolumn{2}{c}{ SEQUENCE } \\
\hline & Apical 4 Chambers & Short Axis \\
\hline Sühling & $0.395 \pm 0.338$ & $0.396 \pm 0.346$ \\
Felsberg & $0.315 \pm 0.257$ & $0.364 \pm 0.293$ \\
Zang & $0.294 \pm 0.217$ & $0.324 \pm 0.256$ \\
Proposed & $\mathbf{0 . 2 6 4} \pm \mathbf{0 . 1 9 0}$ & $\mathbf{0 . 3 1 3} \pm \mathbf{0 . 2 4 2}$ \\
\hline
\end{tabular}

Table II reports the average errors obtained on the entire simulated sequences. For all the algorithms, the parameters have been optimized to obtain the smallest average error on the SAx sequence. For the proposed algorithm these are $\lambda_{0}=2$ and $N_{p}=5$. For the Zang algorithm they are instead $\gamma=0.2$, $\alpha=0.2, \rho=2$ and five pyramidal refinements. The Felsberg algorithm employed a fixed window $w$ given by the tensor product of two B-spline functions of order 5 at scale $J=4$, while the optimal wavelength for the SQF was 3 pixels. The Sühling algorithm employed the multiscale window choice by testing the same scales $j=\{2,3,4,5\}$ as the proposed algorithm. Neither Felsberg's nor Sühling's algorithm applied any refinement scheme like the one in Section III-C (cf. [19], [21]).

From Table II all the three monogenic phase-based algorithms considered perform better than Sühling's algorithm. This confirms that the monogenic phase is a more reliable feature than pixel intensity as far as medical ultrasound is concerned [8], [11], [14]. Also, both the Zang algorithm and the proposed algorithm outperform the Felbsberg algorithm

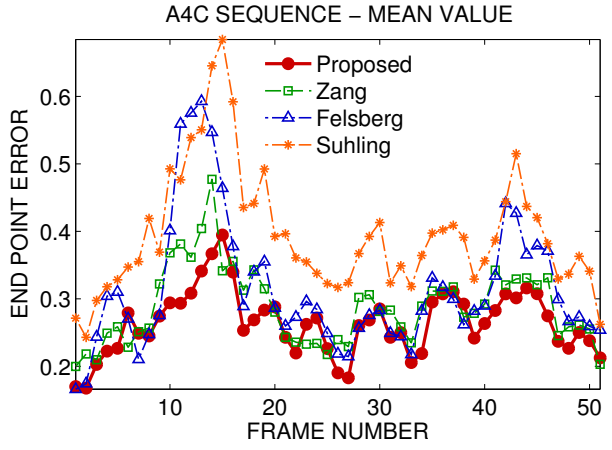

(a)

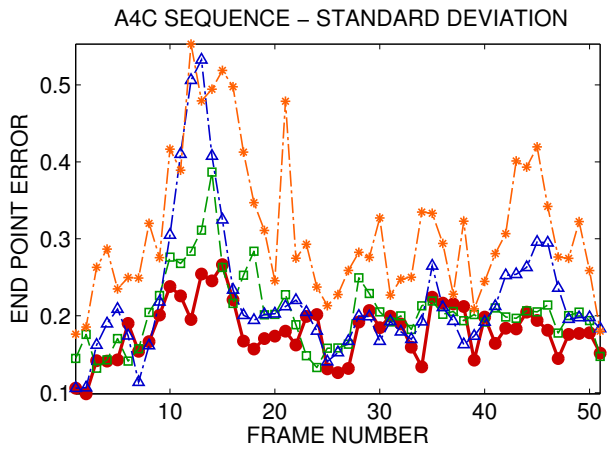

(b)

Fig. 9. Errors for the four algorithm on the synthetic A4C sequence: mean value (a) and standard deviation (b).

due to their more sophisticated formulation. As in the tMRI case, the improvement with respect to the Zang algorithm is less pronounced than with respect to the other two algorithms. Nevertheless, in this case as well, the differences were found to be statistically significant according to a Friedman rank test $(p<0.0001, \alpha=0.05)$.

A more detailed performance analysis is illustrated in Fig. 9, where the four algorithms are compared on the $\mathrm{A} 4 \mathrm{C}$ sequence. The four curves represent the mean value (a) and standard deviation (b) of the endpoint error on each frame of the sequence. As in the tMRI case, the improvement of our algorithm with respect to the Zang algorithm is more relevant for large displacements. In particular, they occur during the diastolic expansion, roughly comprised between frame 10 and frame 22 of the simulated sequence. Again, this superiority can be explained by the major flexibility involved by the proposed formalism, which makes it more suitable for following complex motion patterns. The frames between 22 and 44 instead represent the end of diastole. In this interval, the displacement is minimal and the Fesberg, Zang and the proposed algorithm return close results. Finally, the last frames correspond to the systolic contraction. Here the Zang algorithm and the one proposed herein still give close estimates, while the error for the Felsberg algorithm increases. This flaw results from the absence in the latter of any strategy to account for large displacements, as the pyramidal refinement adopted in the Zang algorithm and the proposed algorithm.

Finally, Fig. 10 shows the benefits derived from the multiscale window choice of Section III-B. The color display 


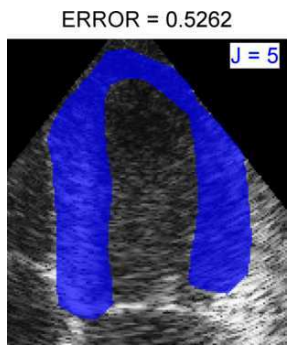

(a)

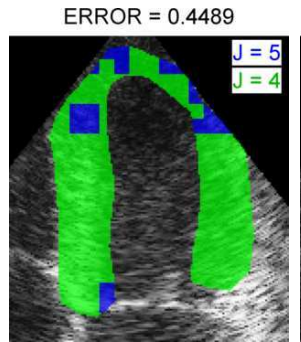

(b)

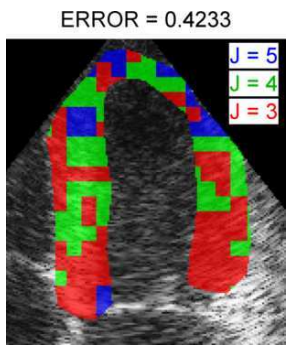

(c)

Fig. 10. Color map illustrating the multiscale window choice. Pixels are colored according to the scale determining their velocity. At the initial step (a) only scale $J=5$ is used, then scale $J=4$ is tested (b) and and the displacements are updated where requested by the error criterion. The window choice procedure ends at scale $J=3$. The title of each figure reports the endpoint error at that step.

represents the scale retained in the velocity computation while the title reports the corresponding endpoint error. The progressive error reduction shows how the window selection procedure allows the computation of more consistent velocity estimates. The block-like appearance of the color maps results from the estimate stopping at scale $j=3$, so that one velocity is computed every $2^{3}$ pixels. A pixel-wise map is then obtained by nearest-neighbor interpolation.

With respect to Fig. 10, it is also interesting to note that, while the scale $j=2$ was also considered, it was never selected in the velocity computation. This reveals that the automatic window selection procedure makes the algorithm almost independent on the chosen range $\left[J_{f}, J_{c}\right]$.

Again, besides being more precise, the proposed algorithm is somewhat more computationally effective than the Zang algorithm. As an example, the computation time for one A4C image (size, $271 \times 333$ pixels $^{2}$ ) with the optimal parameters was $0.68 \mathrm{~s}$ while it was $18.6 \mathrm{~s}$ for the Zang algorithm. This point is even more important here than with MRI. Indeed, although off-line processing is considered acceptable in the latter case, it would not be for ultrasound, where the real-time aspect is one of the major attractions.

\section{CONCLUSION}

We have described a novel algorithm for the analysis of heart motion from medical images. The displacement is estimated from the monogenic phase and is therefore robust to possible variations of the local image energy. A local affine model accounts for the typical contraction, torsion and shear of myocardial fibers. An effective B-spline multiresolution strategy automatically selects the scale returning the most consistent velocity estimate. The multiresolution strategy together with a least-squares estimate of the monogenic orientation make the algorithm robust under image noise.

Due to its general formulation, the proposed algorithm is well suited for measuring myocardial motion from images from different modalities. In particular, we have presented an evaluation on cardiac tagged MRI and echocardiographic sequences. The results have shown that the proposed algorithm is a valid alternative to state-of-the-art techniques in the two fields. Moreover, it was shown to be more accurate and considerably less computation-demanding than another recent algorithm based on the monogenic signal [24].

A potentially valuable application is motion compensation of myocardial perfusion MRI images [7]. Indeed, the major challenge in correcting the motion problem is that the local tissue contrast in the image sequence changes locally with time, especially in the region of interest, the left ventricular myocardium. Due to the low sensitivity to alterations in the brightness profile, we believe the application of the proposed algorithm to this problem could lead to beneficial results.

\section{ACKNOWLEDGMENTS}

This work was partially supported by the US-Tagging grant financed by ANR (Agence Nationale de la Recherche).

\section{APPENDIX A}

\section{THE ZANG ALGORITHM FOR OPTICAL FLOW COMPUTATION}

The Zang algorithm is based on an extension of the monogenic signal for intrinsically 2D structures, called monogenic curvature tensor. The motion estimate is then obtained by plugging this new feature in the popular non-linear energy function of Bruhn et al. [36]:

$$
\begin{array}{r}
E(\mathbf{w})=\int_{\Omega}\left(\psi_{1}\left(\mathbf{w}^{T} J_{\rho}\left(\nabla_{3} \varphi+\gamma \nabla_{3} \Phi\right) \mathbf{w}\right)\right) d x d y+ \\
+\alpha \int_{\Omega} \psi_{2}\left(|\nabla \mathbf{w}|^{2}\right) d x d y .
\end{array}
$$

where $\mathbf{w}=\left[d_{1}, d_{2}, 1\right], \nabla_{3}=\left[\partial_{x}, \partial_{y}, \partial_{t}\right], J_{\rho}\left(\nabla_{3} f\right)=$ $K_{\rho} *\left(\nabla_{3} f \nabla_{3} f^{T}\right), \psi_{i}(z)=2 \beta_{i} \sqrt{1+z / \beta_{i}}, \alpha, \gamma$ and $\beta$ are constant parameters and $K_{\rho}$ is a Gaussian kernel with standard deviation $\rho$. The two terms $\varphi$ and $\Phi$ are the monogenic signal and monogenic curvature phases, respectively.

The minimization of (19) is carried out as in [36] with two nested iterative procedures. An outer fixed point cycle in $\psi_{1}$, $\psi_{2}$ to remove the non-linearity and an inner successive overrelaxation method (SOR) to solve the resulting linear problem. A pyramidal refinement scheme is also employed, as in [36].

\section{REFERENCES}

[1] M. Felsberg and G. Sommer, "The monogenic signal," Signal Processing, IEEE Transactions on, vol. 49, no. 12, pp. $3136-3144$, dec 2001.

[2] J. Noble and D. Boukerroui, "Ultrasound image segmentation: a survey," Medical Imaging, IEEE Transactions on, vol. 25, no. 8, pp. $987-1010$, aug. 2006.

[3] C. Meyer, P. Bland, and J. Pipe, "Retrospective correction of intensity inhomogeneities in mri," Medical Imaging, IEEE Transactions on, vol. 14, no. 1, pp. $36-41$, mar 1995.

[4] L. Axel and L. Dougherty, "MR imaging of motion with spatial modulation of magnetization," Radiology, vol. 171, no. 3, pp. 841-845, Jun. 1989

[5] I. Smal, N. Carranza-Herrezuelo, S. Klein, W. Niessen, and E. Meijering, "Quantitative comparison of tracking methods for motion analysis in tagged mri," in Biomedical Imaging: From Nano to Macro, 2011 IEEE International Symposium on, 30 2011-april 2 2011, pp. 345 -348.

[6] Z. Qian, Q. Liu, D. M., and L. Axel, "Identifying regional cardiac abnormalities from myocardial strains using nontracking-based strain estimation and spatio-temporal tensor analysis," Medical Imaging, IEEE Transactions on, vol. 30, no. 12, pp. 2017 -2029, dec. 2011.

[7] N. M. Wilke, M. Jerosch-Herold, A. Zenovich, and A. E. Stillman, "Magnetic resonance first-pass myocardial perfusion imaging: Clinical validation and future applications," Journal of Magnetic Resonance Imaging, vol. 10, no. 5, pp. 676-685, 1999. 
[8] M. Mellor and M. Brady, "Phase mutual information as a similarity measure for registration," Medical Image Analysis, vol. 9, no. 4, pp. $330-343,2005$.

[9] A. Wong and J. Orchard, "Robust multimodal registration using local phase-coherence representations," J. Signal Process. Syst., vol. 54, no. 1-3, pp. 89-100, Jan. 2009

[10] M. Mulet-Parada and J. Noble, " $2 \mathrm{~d}+\mathrm{t}$ acoustic boundary detection in echocardiography," Medical Image Analysis, vol. 4, no. 1, pp. 21 - 30, 2000.

[11] K. Rajpoot, V. Grau, and J. Noble, "Local-phase based 3d boundary detection using monogenic signal and its application to real-time 3d echocardiography images," in Biomedical Imaging: From Nano to Macro, 2009. ISBI '09. IEEE International Symposium on, 28 2009july 12009 , pp. $783-786$.

[12] A. Belaid, D. Boukerroui, Y. Maingourd, and J.-F. Lerallut, "Phase-based level set segmentation of ultrasound images," Information Technology in Biomedicine, IEEE Transactions on, vol. 15, no. 1, pp. $138-147$, jan. 2011.

[13] V. Grau and J. Noble, "Adaptive multiscale ultrasound compounding using phase information," in Medical Image Computing and ComputerAssisted Intervention MICCAI 2005, ser. Lecture Notes in Computer Science. Springer Berlin / Heidelberg, 2005, vol. 3749, pp. 589-596.

[14] V. Grau, H. Becher, and J. Noble, "Registration of multiview real-time 3-d echocardiographic sequences," Medical Imaging, IEEE Transactions on, vol. 26, no. 9, pp. $1154-1165$, sept. 2007.

[15] N. Chenouard and M. Unser, "3d steerable wavelets and monogenic analysis for bioimaging," in Biomedical Imaging: From Nano to Macro, 2011 IEEE International Symposium on, 30 2011-april 2 2011, pp. 2132 -2135 .

[16] C. Wachinger, T. Klein, and N. Navab, "The 2d analytic signal for envelope detection and feature extraction on ultrasound images," Medical Image Analysis, no. 0, 2012, in press.

[17] M. Sühling, C. Jansen, M. Arigovindan, P. Buser, S. Marsch, M. Unser, and P. Hunziker, "Multiscale Motion Mapping: A Novel Computer Vision Technique for Quantitative, Objective Echocardiographic Motion Measurement Independent of Doppler: First Clinical Description and Validation," Circulation, vol. 110, no. 19, pp. 3093-3099, Nov. 2004.

[18] J. D'hooge, A. Heimdal, F. Jamal, T. Kukulski, B. Bijnens, F. Rademakers, L. Hatle, P. Suetens, and G. R. Sutherland, "Regional strain and strain rate measurements by cardiac ultrasound: Principles, implementation and limitations," Eur. J. Echocardiogr., vol. 1, no. 3, pp. 154-170.

[19] M. Felsberg, "Optical flow estimation from monogenic phase," in Proceedings of the 1st International Conference on Complex motion, ser. IWCM'04, 2004, pp. 1-13.

[20] M. Sühling, M. Arigovindan, P. Hunziker, and M. Unser, "Multiresolution moment filters: theory and applications," Image Processing, IEEE Transactions on, vol. 13, no. 4, pp. 484 -495, april 2004.

[21] M. Sühling, M. Arigovindan, C. Jansen, P. Hunziker, and M. Unser, "Myocardial motion analysis from b-mode echocardiograms," Image Processing, IEEE Transactions on, vol. 14, no. 4, pp. 525 -536, april 2005.

[22] M. Unser, D. Sage, and D. Van De Ville, "Multiresolution monogenic signal analysis using the riesz laplace wavelet transform," Image Processing, IEEE Transactions on, vol. 18, no. 11, pp. $2402-2418$, nov. 2009.

[23] T. Arts, F. Prinzen, T. Delhaas, J. Milles, A. Rossi, and P. Clarysse, "Mapping displacement and deformation of the heart with local sinewave modeling," Medical Imaging, IEEE Transactions on, vol. 29, no. 5, pp. $1114-1123$, may 2010.

[24] D. Zang, L. Wietzke, C. Schmaltz, and G. Sommer, "Dense optical flow estimation from the monogenic curvature tensor," in Proceedings of the 1st international conference on Scale space and variational methods in computer vision, 2007, pp. 239-250.

[25] M. Felsberg and G. Sommer, "The monogenic scale-space: A unifying approach to phase-based image processing in scale-space," Journal of Mathematical Imaging and Vision, vol. 21, pp. 5-26, 2004

[26] G. Granlund and H. Knutsson, Eds., Signal Processing for Computer Vision. Dordrecht: Kluver, 1995.

[27] D. Boukerroui, J. A. Noble, and M. Brady, "On the choice of band-pass quadrature filters," Journal of Mathematical Imaging and Vision, vol. 21, pp. 53-80, 2004.

[28] L. Wietzke and G. Sommer, "The signal multi-vector," Journal of Mathematical Imaging and Vision, vol. 37, pp. 132-150, 2010.

[29] J. Bigun, G. Granlund, and J. Wiklund, "Multidimensional orientation estimation with applications to texture analysis and optical flow," Pattern Analysis and Machine Intelligence, IEEE Transactions on, vol. 13, no. 8, pp. $775-790$, aug 1991.
[30] B. D. Lucas and T. Kanade, "An Iterative Image Registration Technique with an Application to Stereo Vision," in In Proc. Seventh International Joint Conference on Artificial Intelligence, 1981, pp. 674-679.

[31] J. R. Bergen, P. Anandan, K. J. Hanna, and R. Hingorani, "Hierarchical model-based motion estimation," in Proceedings of the Second European Conference on Computer Vision, ser. ECCV '92, 1992, pp. 237-252.

[32] G. Farneback, "Very high accuracy velocity estimation using orientation tensors, parametric motion, and simultaneous segmentation of the motion field," in Computer Vision, 2001. ICCV 2001. Proceedings. Eighth IEEE International Conference on, vol. 1, 2001, pp. 171 -177 vol.1.

[33] M. Ledesma-Carbayo, J. Kybic, M. Desco, A. Santos, M. Suhling, P. Hunziker, and M. Unser, "Spatio-temporal nonrigid registration for ultrasound cardiac motion estimation," IEEE TMI, vol. 24, no. 9, pp. $1113-1126$, sept. 2005.

[34] B. K. P. Horn and B. G. Schunck, "Determining optical flow," Artificial Intelligence, vol. 17, no. 1-3, pp. 185-203, Aug. 1981.

[35] C.-J. Westelius, "Focus of attention and gaze control for robot vision," Ph.D. dissertation, Linköping University, Sweden, SE-581 83 Linköping, Sweden, 1995.

[36] A. Bruhn, J. Weickert, and C. Schnörr, "Lucas/kanade meets horn/schunck: combining local and global optic flow methods," Int. J. Comput. Vision, vol. 61, pp. 211-231, February 2005.

[37] M. Otte and H. Nagel, "Optical flow estimation: Advances and comparisons," in Computer Vision ECCV '94, ser. Lecture Notes in Computer Science, J.-O. Eklundh, Ed. Springer Berlin / Heidelberg, 1994, vol. 800, pp. 49-60.

[38] S. Baker, D. Scharstein, J. P. Lewis, S. Roth, M. J. Black, and R. Szeliski, "A database and evaluation methodology for optical flow," Int. J. Comput. Vision, vol. 92, pp. 1-31, March 2011.

[39] N. Osman, E. McVeigh, and J. Prince, "Imaging heart motion using harmonic phase mri," Medical Imaging, IEEE Transactions on, vol. 19, no. 3, pp. $186-202$, march 2000.

[40] D. J. Fleet and A. D. Jepson, "Computation of component image velocity from local phase information," Int. J. Comput. Vision, vol. 5, no. 1, pp. 77-104, Sep. 1990.

[41] P. Clarysse, J. Tafazzoli, P. Delachartre, and P. Croisille, "Simulation based evaluation of cardiac motion estimation methods in tagged-mr image sequences," Journal of Cardiovascular Magnetic Resonance, vol. 13, p. 360, feb 2011.

[42] P. Clarysse, C. Basset, L. Khouas, P. Croisille, D. Friboulet, C. Odet, and I. Magnin, "2d spatial and temporal displacement field fitting from cardiac mr tagging," Medical Image Analysis, vol. 3, pp. 253-268, 2000.

[43] W. Daniel, Applied Nonparametric Statistics. Duxbury Thomson Learning, 2000.

[44] V. Chalana and Y. Kim, "A methodology for evaluation of boundary detection algorithms on medical images," Medical Imaging, IEEE Transactions on, vol. 16, no. 5, pp. 642 -652, oct. 1997.

[45] B. Galvin, B. Mccane, K. Novins, D. Mason, and S. Mills, "Recovering motion fields: An evaluation of eight optical flow algorithms," in British Machine Vision Conference, 1998, pp. 195-204.

[46] T. L. Szabo, Diagnostic Ultrasound Imaging: Inside Out. Burlington , MA: Elsevier Academic Press, 2004.

[47] G. R. Sutherland, G. D. Salvo, P. Claus, J. D'hooge, and B. Bijnens, "Strain and strain rate imaging: a new clinical approach to quantifying regional myocardial function," Journal of the American Society of Echocardiography, vol. 17, no. 7, pp. 788 - 802, 2004.

[48] T. Marwick, M. Yu, and J. Sun, Myocardial Imaging: Tissue Doppler and Speckle Tracking. Carlton: Blackwell Science Asia, 2007.

[49] J. Kybic and M. Unser, "Fast parametric elastic image registration," IEEE TIP, vol. 12, no. 11, pp. 1427 - 1442, nov. 2003.

[50] T. Maltaverne, P. Delachartre, and A. Basarab, "Motion estimation using the monogenic signal applied to ultrasound elastography," in Engineering in Medicine and Biology Society (EMBC), 2010 Annual International Conference of the IEEE, 31 2010-sept. 4 2010, pp. 33 -36 .

[51] M. Alessandrini, H. Liebgott, D. Friboulet, and O. Bernard, "Simulation of realistic echocardiographic sequences for ground truth validation of motion estimation," in IEEE International Conference on Image Processing (ICIP), Orlando, USA, 2012, accepted.

[52] T. Dietenbeck, M. Alessandrini, D. Barbosa, J. D'hooge, D. Friboulet, and O. Bernard, "Detection of the whole myocardium in $2 \mathrm{~d}$ echocardiography for multiple orientations using a geometrically constrained level-set," Medical Image Analysis, vol. 16, no. 2, pp. $386-$ 401, 2012. 